\title{
TRANSFUSIONS OF SUSPENSIONS OF BLOOD PLATELETS IN THROMBOCYTOPENIA AND THROMBOPATHIA
}

\author{
BY \\ S. VAN CREVEld, M. M. P. PAUlSSEN, AND H. L. J. M. BARTELS \\ with the technical assistance of \\ R. VONK \\ From the Paediatric Clinic, University of Amsterdam, The Netherlands
}

(RECEIVED FOR PUBLICATION OCTOBER 10, 1952)

In the treatment of the different syndromes which are accompanied by thrombocytopenia blood transfusions are usually given. Of late, some investigators have used blood very rich in blood platelets, namely, blood of patients suffering from polycythaemia (Stefanini, Chatterjea, Dameshek, Zannos, and Perez Santiago, 1952). The possibility of treating thrombocytopenia by means of suspensions of thrombocytes has been considered (Dillard, Brecher, and Cronkite, 1951 ; Juillard, Maupin, Loverdo, Bernard, Colvez, and Lecomte, 1952 ; Minor and Burnett, 1952), but the majority were of the opinion that thrombocytes outside their natural medium of plasma are too unstable.

The results obtained with transfusions of normal blood are generally unsatisfactory. One of the explanations of this fact is that the treatment of the blood before the transfusion does not usually guarantee that the thrombocytes are not destroyed before the transfusion or that their functions remain more or less normal. The technique with silicone (Jaques, Fidlar, Felsted, and Macdonald, 1946) theoretically overcomes part of these difficulties. but with this technique the objection remains that too large a quantity of normal blood is necessary to influence an existing thrombocytopenia adequately. The use of blood of patients with polycythaemia, of which good results have been communicated recently, has the great drawback that these patients are few.

We have examined the effect of the intravenous injection of concentrated suspensions of platelets in different conditions of thrombocytopenia and also in some cases of thrombopathia. In preparing platelet suspensions we have used a centrifugation and resuspension technique rather different from that used earlier by other authors (Copley and Houlihan, 1947 ; Ware, Fahey, and Seegers, 1948 ; Zucker, 1951).

\section{Preparation of the Suspension of Blood Platelets}

Blood, $500 \mathrm{ml}$, obtained by venepuncture, is collected in a sterile siliconized bottle, cooled to $0-1^{\circ} \mathrm{C}$., which contains $90 \mathrm{ml}$. of a mixture of citrate and glucose $(3.3 \%$ disodium citrate and $3.3 \%$ glucose). The blood is then poured into sterile, cooled, and siliconized bottles of $250-\mathrm{ml}$. capacity closed by means of cotton-wool covered with cellophane, and centrifuged for 15 minutes at 1,500 r.p.m. at a temperature of $0-1^{\circ}$ C. (M.S.E. refrigeratorcentrifuge). The supernatant plasma, which is rich in platelets, is removed with a sterile syringe and thick needle or a sterile siphon and put into cooled siliconized tubes. Eventually the remaining blood is once more centrifuged in the same way and the plasma collected. The plasma is then centrifuged for 30 minutes at $0-1^{\circ} \mathrm{C}$. at 3,000 r.p.m. The supernatant plasma is pipetted or sucked off. A white, rather compact layer of thrombocytes remains on the bottom of the tubes, generally containing some leucocytes and few red blood cells. To this layer is added $10-20 \mathrm{ml}$. of a cooled $\left(0-1^{\circ} \mathrm{C}\right.$.) mixture of sodium chloride/sodium citrate $(9$ parts $\mathrm{NaCl} 0.9 \%$ to 1 part disodium citrate $3.3 \%$ ). With a sterile siliconized glass rod the layer of thrombocytes is loosened from the bottom, and then in the course of one to two hours at $10-12^{\circ} \mathrm{C}$. these thrombocytes spontaneously become suspended in the fluid. Sometimes occasional slight shaking is necessary. This homogeneous suspension, after remaining for a very short time in a water bath at $37^{\circ} \mathrm{C}$., is injected slowly into a vein. Special care is taken to prevent blood from the vein from running into the suspension, because there is a risk that coagulation may begin in the syringe, thus destroying thrombocytes.

We have repeatedly compared the quantity of blood platelets in the withdrawn blood with the 
quantity in the suspension. It appeared that with our method $60-75^{\circ}$ of the thrombocytes remained in the suspension. Moreover the lost thrombocytes need not be destroyed but for the greater part are still present between the erythrocytes. We did not take the trouble to free the suspension of thrombocytes completely of erythrocytes or leucocytes, as this purification would cause too big a loss of blood platelets. As a precaution and also as the antigenic structure of the thrombocytes is unknown, we always started with blood from the same ABO and $\mathrm{Rh}$ blood group as those of the patient. The quantity of erythrocytes in the suspension, however, was minimal (about 1 per 1,000 thrombocytes); the suspension was milky white.

The morphological aspect of the thrombocytes in the fresh suspension was normal. They looked like flat, round discs. Agglutination of platelets in the suspension was seen only once and in this case the suspension was naturally not injected. Apparently the suspension remains unchanged for some days provided that it is kept at $4^{\circ} \mathrm{C}$. For transfusion of we always made use of a freshly prepared suspension of thrombocytes counted after the method of Rees and Ecker (1923); the clot retraction was determined by Macfarlane's method (1939).

The preliminary, hopeful results of the transfusion $\stackrel{\mathbb{Q}}{\varrho}$ of suspensions of thrombocytes into a number of $๗$ patients with thrombocytopenia and in two patients $\vec{O}$ with thrombopathia are now recorded.

\section{Case Reports}

Case 1 : Congenital Thrombocytopenia.-This child, R. K., born in November, 1949, has been described in detail by Stoppelman and Blaauw-van Dok (1950). As well as having functional disorders of the bone $\nexists$ marrow he was also suffering from toxoplasmosis. $\vec{O}$ Immediately after birth, which was spontaneous at full term (birth weight $2,650 \mathrm{~g}$.), the child showed large and small haemorrhages of the skin all over $\mathbb{D}$

TABLE I

RESULTS OF TRANSFUSIONS OF SUSPENSIONS OF THROMBOCYTES IN NINE CASES




the body and over the greater part of the head. In the occipital region there was a very large haemorrhage. The child vomited bloody fluid several times. The peripheral blood showed a leucopenia $(2,200)$. The haemoglobin content was $110 \%$, the erythrocyte count 5,600,000 (thrombocytes, 0.6000). The bleeding time was prolonged. The bone marrow was poor in cells, contained many lymphocytes, and only a few leucocytes of the myeloid series. Only two megakaryocytes could be found.

Before admission to the Children's Clinic on the tenth day of life the child had already had two blood transfusions without any influence upon the leucopenia and thrombopenia. We gave him several transfusions of suspensions of thrombocytes. The concentration of the suspension used in the first transfusion was unknown. The influence of these suspensions on the number of blood platelets can be seen from Table I, from which it appears that running parallel to the transfusions of platelets the thrombocyte count of the patient increased, an augmentation which was maintained after the fifth transfusion. The disturbed clot retraction also became normal. At the age of 3 months for the first time the bone marrow showed a satisfactory number of megakaryocytes, so that we could make a favourable prognosis.

When 5 months old the child's blood contained 230,000 thrombocytes per $\mathrm{mm}^{3}$

Case 2 : Congenital Thrombocytopenia.-F. J. V. was a full-term child, born spontaneously with a birth weight of $3,000 \mathrm{~g}$., the first child of healthy parents. At the age of 2 months the child had an epistaxis. At the age of 4 months the whole body was covered with petechiae, with an occasional larger haemorrhage. The liver became somewhat enlarged; the spleen was palpable half a fingerbreadth below the costal margin. A tourniquet test was positive. In the peripheral blood not a single thrombocyte could be found: the haemoglobin content was $60 \%$, the erythrocyte count $3,500,000$ per $\mathrm{mm}^{3}$, the leucocyte count 10,000 ( $8 \%$ eosinophils). The bleeding time was prolonged and clot retraction was poor. The bone marrow was rich in cells and contained a large number of megakaryocytes.

At the age of 4 months the child received a transfusion of a suspension of thrombocytes (Table I). One hour later the platelet count had only slightly risen and remained about the same for four days; during this time the number of petechiae diminished distinctly. Five days after transfusion the face, hands, and feet were free from haemorrhages. In the following days the child received a further two transfusions of a platelet suspension. By these transfusions the platelet count increased only slightly, but the number of petechiae decreased. Five days after the last transfusion the platelet count was almost nil and new petechiae appeared. A repeated transfusion of fresh citrated blood was not followed by a rise of the thrombocyte count. Thus in this case of congenital thrombocytopenia the transfusions of suspensions of thrombocytes had only a moderate success. Later on there appeared in this child, as often happens in analogous cases, a spontaneous rise of the thrombocyte count.

Case 3: Essential Thrombocytopenia.-Since the age of 4 years this girl, J. S., has regularly had blue spots all over the body and from the age of $4 \frac{1}{2}$ years she has also had an occasional epistaxis. As these were increasing in frequency she was admitted to hospital when $5 \frac{1}{2}$ years old. No abnormalities were noted in the thorax and abdomen. The blood was characteristic of essential thrombocytopenia. The bone marrow was rich in cells and active, the number of megakaryocytes was adequate, but the protoplasm was not granular, and the formation of thrombocytes was deficient (Dr. S. I. de Vries).

After the patient's discharge from hospital the thrombocytopenia continued for two years with a thrombocyte count which at various times was never higher than 70,000 per $\mathrm{mm}^{3}$ (usually between 30,000 and 50,000 per $\mathrm{mm}^{3}$ ).

When admitted to the Children's Clinic on June 25, 1952 , the child was $6 \frac{1}{2}$ years old. The diagnosis was essential thrombocytopenia, and the thrombocyte count 35,000 per $\mathrm{mm}^{3}$

On June 26, July 3, and July 10, 1952, the child received three transfusions of a suspension of thrombocytes (Table I). After every transfusion of platelets the thrombocyte count rose significantly and after the third transfusion was as high as 243,000 per $\mathrm{mm}^{3}$ The influence of the first transfusion was maintained for seven days; the influence of the second and third transfusions lasted for a shorter period, but after both transfusions of platelets a secondary rise in the thrombocyte count occurred.

Parallel with the rise in the thrombocyte count the clot retraction, determined by Macfarlane's (1939) method, also increased, while the activity of neutralizing heparin became greater.

Case 4 : Acute Leukaemia with Thrombocytopenia. -B. W., a $5 \frac{1}{2}$-year-old boy, received three transfusions of suspensions of thrombocytes: The changes in the platelet count, in the clot retraction, and in the bleeding time, determined by Duke's method, following these transfusions are given in Table $I$. The effect of each of the transfusions lasted some days. Seven days after the last transfusion, however, a spontaneous rise in the thrombocyte count occurred, and this soon reached a normal value which was maintained for some weeks; thereafter the platelet count decreased.

Case 5 : Acute Leukaemia with Thrombocytopenia. -A. S., a 3-year-old girl, was given seven transfusions of suspensions of platelets, the results of which are given in Table I. After each transfusion a transitory increase of the thrombocyte count took place and at the same time a decrease in the bleeding time and an increase in the clot retraction. The effect of each transfusion lasted for two to four days. 
Case 6: Aplastic Aleukaemic Leukaemia with Thrombocytopenia.-H. E., a boy aged 10 years, received three transfusions of suspensions of platelets, the results of which are given in Table I. After the first transfusion there was only a very small rise of the number of platelets; after the second transfusion the number of platelets showed a distinct increase, which slowly disappeared. Before the number of platelets had returned to the original level a new transfusion was given with a slight and temporary effect.

Case 7 : Chronic Myeloid Leukaemia in an Aplastic Stage.-G. W., a 13-year-old girl, received four transfusions of suspensions of platelets. The effect of each transfusion was only slight and had always disappeared one hour after the transfusion (compare the results of Stefanini et al., 1952).

Case 8 : Thrombopathia.-C. W., a 10-year-old girl, has been described in an earlier paper (Van Creveld and Paulssen, 1951) as suffering from a haemorrhagic diathesis with a normal platelet count but with a dysfunction of the platelets as regards the neutralization of added heparin, which we ascribed to a deficiency of factor 3 . The clot retraction was normal. In this patient we investigated the effect of a transfusion of a suspension of platelets upon the ability of the blood to neutralize heparin. From Fig. 1 it

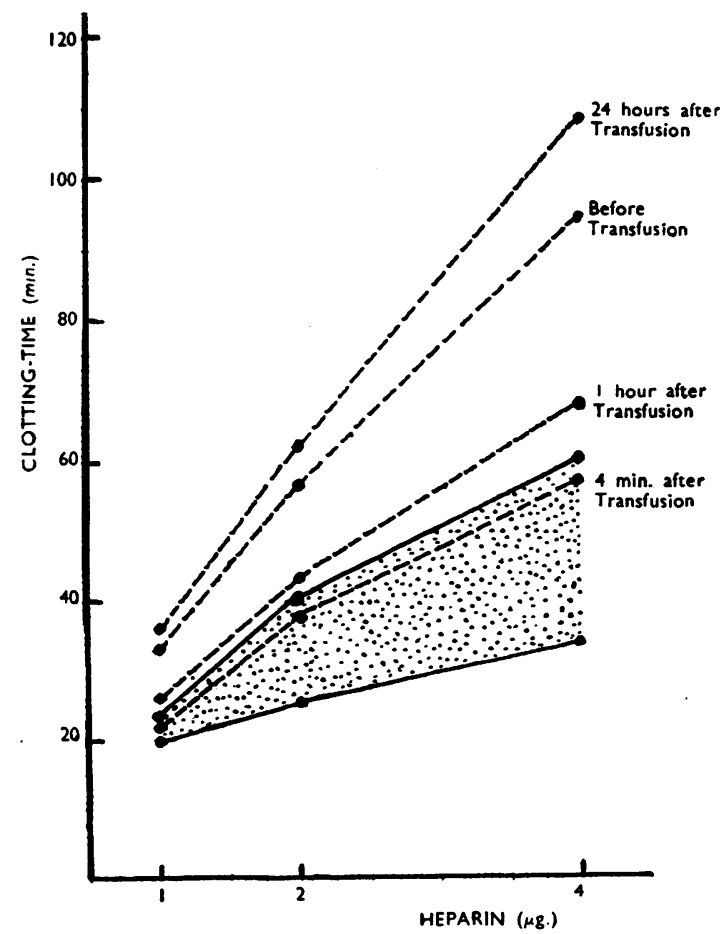

FIG. 1.-Anti-heparin activity of blood in a child with thrombopathia (Case 8) before and at different times (four minutes, one hour, 24 hours) after transfusion of a suspens
platelets. Shaded area indicates normal range. can be seen that after a transfusion the ability to neutralize added heparin returned to normal for a short time.

Case 9 : Thrombasthenia (Glanzmann). - C. S., a 13-든 year-old girl, had a normal platelet count, but the clot $\frac{\overline{\bar{F}}}{\frac{\mathrm{F}}{\mathrm{S}}}$ retraction was disturbed and the bleeding time greatly $\overparen{\nabla}$ prolonged. The ability to neutralize heparin was normal. This patient received three transfusions of $\infty$ a suspension of platelets. The results (Table I) $\vec{\circ}$ show that after each transfusion the bleeding time and the clot retraction were favourably influenced $\vec{\omega}$ temporarily.

\section{Discussion}

To these preliminary results we can add the fol- lowing. None of our patients showed after one or $\vec{A}$ repeated transfusions of suspensions of platelets음 any subjective or objective disagreeable symptom.The conclusion seems justified that at the moment $T$ when the platelets were injected these for the $\bar{D}$ greater part at least had not lost their function. We deduce this from the fact that in the various patients symptoms of a deficiency or an inferiority $\vec{\varphi}$ of platelets (platelet count, clot retraction, bleeding time) were temporarily relieved by the transfusion. This fact points to the possibility of using a trans-O fusion of platelets therapeutically for haemorrhages in thrombocytopenia or thrombopathia and as prophylaxis against haemorrhages in such con- $\frac{2}{\Phi}$ ditions.

We would stress the remissions observed in Cases $\overrightarrow{\vec{O}}$ 1 and 3, and perhaps also in Case 4 , after trans- 3 fusions of suspensions of platelets. These remissions occurred shortly after the rise of platelets, caused by the transfusion, had gradually용 diminished.

In the paper by Stefanini et al. (1952) we found that one of these authors had not infrequently? observed an analogous remission after transfusions of blood rich in platelets in patients with idio-o pathic thrombocytopenic purpura. Our experiences might indicate that the remission in theseo cases is due to the transfusion, especially of the platelets, and that it has a stimulating influence ono the new production of platelets.

\section{Summary}

This communication describes the resultso obtained in a number of children with primary or secondary thrombocytopenia and in two chil-? dren with thrombopathia who all received repeated intravenous injections of suspensions of thrombo- $\frac{\text { a }}{8}$. cytes. A description of the techniques of prepar ing the suspensions of platelets is given. The changes in the platelet count were closely paralleledo by changes in clot retraction and bleeding time 
Three of the eight patients after the transfusions of platelets showed an important rise in the thrombocyte count. In two cases of primary thrombocytopenia the rise has been maintained.

This investigation was made possible by the Netherlands Organization for Pure Research (Z.W.O.) and by the Netherlands Organization for Applied Scientific Research (T.N.O.).

We are indebted to Professor J. G. G. Borst and Dr. J. A. Molhuysen from the University Clinic for Internal Diseases for their help in obtaining blood from patients with polycythaemia.
REFERENCES

Copley, A. L., and Houlihan, R. B. (1947) Blood, Special Issue, 1,182

Creveld, S. van, and Paulssen, M. M. P. (1951). Lancet, 2, 242.

Dillard, G. H. L., Brecher, G., and Cronkite, E. P. (1951). Proc. Soc. exp. Biol. N.Y., 78, 796.

Jaques, L. Fidlar, E., Feldsted, E. T., and Macdonald, A. G. (1946). Canad med. Ass. J., 55, 26.

Julliard, J., Maupin, B., Loverdo, A., Bernard, J., Colvez, P., and Lecomte, M. (1952). Presse med., 60, 518 .

Macfarlane, R. G. (1939). Lancet, 1, 1199 .

Minor, A. H., and Burnett, L. (1952). Blood, 7, 693.

Rees, H. M., and Ecker, E. E. (1923). J. Amer. med. Ass., 80, 621.

Stefanini, M., Chatterjea, J. B. Dameshek, W., Zannos, L., and Perez M., Chatterjea, J. B., Dameshek, W., Zannos, L., and Perez Santiago, E. P. (1952). Ibid., 7, 53 .

Stoppelman, M. R. H., and Blaauw-van Dok, S. (1950). Ned. T. Geneesk., 94, 1374.

Ware, A. G., Fahey, J. L., and Seegers, W. H. (1948). Amer. J. Physiol., 154, 140.

Zucker, M. B. (1951). Blood Clotting and Allied Problems: Transactions of the Fou th Conference, Josiah Macy, Jr., Foundation, p. 143. New York. 